\title{
A Brief Note Concerning Non-Self Contractions in Banach Spaces Endowed with a Graph
}

\author{
Esra Yolacan \\ Republic of Turkey Ministry of National Education, 60000 Tokat-Turkey \\ yolacanesra@gmail.com
}

\begin{abstract}
In this paper, we establish the concept of non-self Hardy-Rogers contractions on Banach spaces involving a graph. We also prove some fixed point theorems for such mappings. The results of this paper extend and generalize various known results in the literature.
\end{abstract}

Keywords: Banach space, $G$-contaction, Rothe's baundary condition, property $(M)$, non-self maping. MSC2010 47H09, 47H10, 54H25.

\section{Introduction and Preliminaries}

Let $M$ be a non-empty set. Given a positive integer $n$, let $M^{n}$ be the product space $M \times M \times \cdots \times$. Let $\mathbb{N}=\{0,1,2, \cdots\}$ be the set of all nonnegative integers. Unless indicated otherwise, "for all $n \geq 1$ " will stand for "for all $n "$.

The proverbial Banach contraction principle (briefly, BCP) is one of the most crucial results in fixed point theory. In the setting of metric space it can be tersely expressed as follows.

[18] If $(M, d)$ is a complete metric space and $g: M \rightarrow M$ is a self mapping such that

$$
d(g x, g y) \leq a d(x, y), \text { for all } x, y \in M,
$$

where $a \in[0,1)$, then $f$ has a unique fixed point.

This principle guarantees the existence and uniqueness of fixed points of self mappings in metric spaces and it also favour with an advantageous constructive procedure to obtain those fixed points.

Jachymski (2008) 1 flourish the BCP for mappings on a metric space involving with a graph. The author 1 proved that Ran and Reurings [20] and Edelstein [21] are obtained by [1. Afterwards, several articles which deal with fixed point theorems for single valued and multivalued mappings in complete metric space with a directed graph appeared [[2]-[10]], [[15]-[17]].

Let $(M, d)$ be a metric space, $\Delta$ be a diagonal of $M^{2}$, and $G$ be a directed graph with no parallel edges such that the set $V(G)$ of its vertices coincides with the points of $M$ and $\Delta \subset E(G)$, where $E(G)$ is the set of the edges of the graph. Then, $G=(V(G), E(G))$. The conversion of a graph $G$ is the graph obtained from $G$ by reversing the direction of edges indicated by $G^{-1}$, i.e., $E\left(G^{-1}\right)=\left\{(x, y) \in M^{2}:(y, x) \in E(G)\right\}$. If $x$ and $y$ be vertices of a graph $G$, then a path in $G$ from $x$ to $y$ of length $\mathbb{N}$ is a sequence $\left\{x_{i}\right\}_{i=0}^{N}$ of $N+1$ vertices such that $x_{0}=x, x_{N}=y$ and $\left(x_{i-1}, x_{i}\right) \in E(G)$ for $i=1, \ldots, N$. A graph $G$ is connected if there is a path between any two vertices. If $G=(V(G), E(G))$ is a graph and $A \subset V(G)$, then the graph $(A, E(A))$ with $E(A)=E(G) \cap\left(A^{2}\right)$ is called the subgraph of $G$ determined by $A$. Denoted by $G_{A}$. If $\tilde{G}=(M, E(\tilde{G}))$ is the symmetric graph obtained by putting together the vertices of both $G$ and $\tilde{G}$, i.e., $E(\tilde{G})=E(G) \cup E\left(G^{-1}\right)$, then $G$ is called weakly connected if $\tilde{G}$ is connected. 
[1] Let $(M, d)$ be a metric space. (i) We say that a mapping $g: M \rightarrow M$ is (well) defined, if it has the property

$$
\forall x, y \in M,(x, y) \in E(G) \Rightarrow(g x, g y) \in E(G) .
$$

(ii) We say that a mapping $g: M \rightarrow M$ is $G$-contraction if $g$ is (well) defined and there exists $\alpha_{1} \in[0,1)$ such that

$$
\forall x, y \in M,(x, y) \in E(G) \Rightarrow d(f x, f y) \leq \alpha_{1} d(x, y) .
$$

(iii) We say that a mapping $g: M \rightarrow M$ is orbitally $G$-continuity if $\forall x, y \in M$ and any sequence $\left\{k_{n}\right\}_{n \in \mathbb{N}}$ of positive integers,

$$
g^{k_{n}} x \rightarrow y,\left(g^{k_{n}} x, g^{k_{n+1}} x\right) \in E(G) \Rightarrow g\left(g^{k_{n}} x\right) \rightarrow g y .
$$

Let $M$ be a Banach space, $H$ a non-empty closed of $M$ and $g: H \rightarrow M$ a non-self mapping. If $x \in H$ is such that $g x \notin H$, then we can always select an $y \in \partial H$ (the boundary of $H$ ) such that $y=(1-\beta) x+\beta g x(\beta \in(0,1)$ ), which virtually state that

$$
d(x, g x)=d(x, y)+d(y, g x), y \in \partial H,
$$

where $d(x, y)=\|x-y\|$.

The set $N$ of points $y$ providing (4) may involve more than one element. Assume that $N$ is non-empty.

14. Let $M$ be a Banach space, $H$ a non-empty closed of $M$ and $g: H \rightarrow M$ a non-self mapping. Let $x \in H$ with $g x \notin H$ and let $y \in \partial H$ be the corresponding elements given by (4). If, for any such elements $x$, we have

$$
d(y, g y) \leq d(x, g x)
$$

for all corresponding $y \in N$, then we say that $g$ has property $(M)$.

[1] Let $M$ be a Banach space, $H$ a non-empty closed of $M$. (i) We say that a mapping $g: H \rightarrow M$ is (well) defined, if it has the property for the subgraph of $G$ induced by $H$, i.e,

$$
\forall x, y \in H,(x, y) \in E(G) \text { with } g x, g y \in H \Rightarrow(g x, g y) \in E(G) \cap\left(H^{2}\right) .
$$

(ii) We say that a mapping $g: H \rightarrow M$ is $G_{H}$-contraction if $g$ is (well) defined and there exists $\alpha_{2} \in[0,1$ ) such that

$$
\forall x, y \in H,(x, y) \in E\left(G_{H}\right) \Rightarrow d(g x, g y) \leq \alpha_{2} d(x, y) .
$$

[1] (Property $(L))$ Any sequence $\left\{x_{n}\right\}_{i=1}^{\infty} \subset M$ with $x_{n} \rightarrow x$ as $n \rightarrow \infty$ and $\left(x_{n}, x_{n+1}\right) \in E(G)$ for all $n \in \mathbb{N}$, there exists a subsequence $\left\{x_{k_{n}}\right\}_{i=1}^{\infty}$ satisfying $\left(x_{k_{n}}, x\right) \in E(G)$ for all $n$.

On the other hand, Berinde and P $\breve{a}$ curar (2015) [11] defined the notion of $G_{H}$-contractions in Banach space with a graph and subsequently presented some fixed point results for such classes of non-self contractions. Balog and Berinde (2016) [12] introduced the concept of $G_{H}$-Kannan contractions on Banach space endowed with a graph and their results extended and generalized the authors [1]. In the sequel, Balog et al. [13. showed some fixed point theorems for $G_{H}$-Catterjea contractions in Banach space involving a graph. They also have generalized the above-mentioned papers.

Motivated by previous results, we establish the concept of non-self Hardy-Rogers contractions on Banach spaces with a graph. We also prove some fixed point theorems for such mappings. The results of this paper extend and generalize various known results in the literature.

\section{Main Results}

Let $(M, d, G)$ be a Banach spaces involving a simple directed and weakly connected graph $G$ such that the property $(L)$ holds. Let $H$ be a non-empty closed of $M$ and $g: H \rightarrow M$ be a $G_{H}$ - Hardy-Rogers contraction with property $(M)$, that is, there exist constants $a, b, c \geq 0$ with $a+2 b+2 c<1$ such that

$$
d(g x, g y) \leq a d(x, y)+b[d(x, g x)+d(y, g y)]+c[d(x, g y)+d(y, g x)],
$$

for all $(x, y) \in E\left(G_{H}\right)$, where $G_{H}$ is the subgraph of $G$ determined by $H$. If $H_{g}:=\{x \in \partial H:(x, g x) \in E(G)\} \neq \emptyset$ and $g$ satisfies Rothe's baundary condition

$$
g(\partial H) \subset H
$$

then $g$ has a fixed point in $H$. 
Proof. Take $g(C) \subset C$, then $g$ is basically a self-map of $H$ and the consequence follows by [19] with $M=H$. Thus, we just take into account $g(H) \nsubseteq H$. Let $x_{0} \in H_{g}$ such that $\left(x_{0}, g x_{0}\right) \in E(G)$. Using (1), we get

$$
\left(g x_{0}, g^{n+1} x_{0}\right) \in E(G) \text { for all } n .
$$

Denote $y_{n}:=g^{n} x_{0}$, for all $n \in \mathbb{N}$. By Rothe's baundary condition, we have $g x_{0} \in H$.

Denote $x_{1}:=g x_{0}=y_{1}$. Next, if $g x_{1} \in H$, set $x_{2}:=g x_{1}=y_{2}$. If $g x_{1} \notin H$, we can select an element $x_{2}$ on the segment $\left[x_{1}, g x_{1}\right]$ which also appertain to $\partial H$, in other words, $y=(1-\beta) x+\beta g x(\beta \in(0,1))$. Continuing in this manner we constitute two sequences $\left\{x_{n}\right\}$ and $\left\{y_{n}\right\}$ whose terms satisfy one of the following specifications:

1. $g x_{n-1} \in H \Rightarrow x_{n}:=g x_{n-1}=y_{n}$;

2. $g x_{n-1} \notin H \Rightarrow x_{n}=(1-\beta) x_{n-1}+\beta g x_{n-1} \in \partial H(\beta \in(0,1))$.

Now, we assert that $\left\{x_{n}\right\}$ is a Cauchy sequence. Suppose that

$$
\begin{gathered}
P=\left\{x_{k} \in\left\{x_{n}\right\}: x_{k}=g x_{k-1}=y_{k}\right\}, \\
Q=\left\{x_{k} \in\left\{x_{n}\right\}: x_{k} \neq g x_{k-1}\right\} .
\end{gathered}
$$

Openly, if $x_{k} \in Q$, then $x_{k-1}$ and $x_{k+1}$ appertain to $P$. Further, by Rothe's baundary condition, we cannot get two successive terms of $\left\{x_{n}\right\}$ in $Q$ (yet we can get two successive terms of $\left\{x_{n}\right\}$ in $P$ ). Now, we deduce that there are the following possibilities.

Case 1. Let $x_{n}, x_{n+1} \in P$, then $x_{n}=g x_{n-1}=y_{n}, x_{n+1}=g x_{n}=y_{n+1}$. Therefore

$$
d\left(x_{n+1}, x_{n}\right)=d\left(g x_{n}, g x_{n-1}\right)=d\left(y_{n+1}, y_{n}\right) .
$$

Due to the fact that $\left\{x_{n}\right\} \subset H$ for all $n$, by $\left(x_{n}, x_{n-1}\right) \in E\left(G_{H}\right)$, and so by $G_{H}-$ Hardy-Rogers contraction condition (8), we get that

$$
\begin{aligned}
d\left(x_{n+1}, x_{n}\right)= & d\left(g x_{n}, g x_{n-1}\right) \\
\leq & a d\left(x_{n}, x_{n-1}\right)+b\left[d\left(x_{n}, g x_{n}\right)+d\left(x_{n-1}, g x_{n-1}\right)\right] \\
& +c\left[d\left(x_{n}, g x_{n-1}\right)+d\left(x_{n-1}, g x_{n}\right)\right]
\end{aligned}
$$

by rearranging,

$$
d\left(x_{n+1}, x_{n}\right) \leq \eta d\left(x_{n-1}, x_{n}\right)
$$

where $\eta=\frac{a+b+c}{1-b-c}<1$.

Case 2. Let $x_{n} \in P, x_{n+1} \in Q$, then $x_{n}=y_{n}, x_{n+1} \neq y_{n+1}$. By $G_{H}$ - Hardy-Rogers contraction condition (8), We get

$$
\begin{aligned}
& d\left(x_{n}, x_{n+1}\right) \\
\leq & d\left(x_{n}, x_{n+1}\right)+d\left(x_{n+1}, y_{n+1}\right) \\
= & d\left(x_{n}, y_{n+1}\right)=d\left(y_{n}, y_{n+1}\right)=d\left(x_{n}, g x_{n}\right) \\
= & d\left(g x_{n-1}, g x_{n}\right) \quad\left(\operatorname{by}\left(x_{n-1}, x_{n}\right) \in E\left(G_{H}\right)\right) \\
\leq & a d\left(x_{n-1}, x_{n}\right)+b\left[d\left(x_{n-1}, g x_{n-1}\right)+d\left(x_{n}, g x_{n}\right)\right] \\
& +c\left[d\left(x_{n-1}, g x_{n}\right)+d\left(x_{n}, g x_{n-1}\right)\right]
\end{aligned}
$$

which yields

$$
d\left(x_{n}, g x_{n}\right) \leq \eta d\left(x_{n-1}, x_{n}\right)
$$

Thus,

$$
d\left(x_{n}, x_{n+1}\right) \leq d\left(x_{n}, g x_{n}\right) \leq \eta d\left(x_{n-1}, x_{n}\right) .
$$

Case 3. Let $x_{n} \in Q, x_{n+1} \in P$, then $x_{n+1}=g x_{n}, y_{n} \neq x_{n}$ and

$$
d\left(x_{n-1}, x_{n}\right)+d\left(x_{n}, g x_{n-1}\right)=d\left(x_{n-1}, g x_{n-1}\right),
$$


from the property $(M)$, we have

$$
\begin{aligned}
d\left(x_{n}, x_{n+1}\right)= & d\left(x_{n}, g x_{n}\right) \leq d\left(x_{n-1}, g x_{n-1}\right)=d\left(g x_{n-2}, g x_{n-1}\right) \\
x_{n} \in & \left.Q \Rightarrow x_{n-1} \in P \quad \text { by }\left(x_{n-2}, x_{n-1}\right) \in E\left(G_{H}\right) \text { and (8) }\right) \\
d\left(x_{n}, x_{n+1}\right) \leq & a d\left(x_{n-2}, x_{n-1}\right)+b\left[d\left(x_{n-2}, g x_{n-2}\right)+d\left(x_{n-1}, g x_{n-1}\right)\right] \\
& +c\left[d\left(x_{n-2}, g x_{n-1}\right)+d\left(x_{n-1}, g x_{n-2}\right)\right] \\
\leq & a d\left(x_{n-2}, x_{n-1}\right)+b\left[d\left(x_{n-2}, x_{n-1}\right)+d\left(x_{n-1}, g x_{n-1}\right)\right] \\
& +c\left[d\left(x_{n-2}, x_{n-1}\right)+d\left(x_{n-1}, g x_{n-1}\right)\right]
\end{aligned}
$$

which yields

$$
d\left(x_{n-1}, g x_{n-1}\right) \leq \frac{a+b+c}{1-b-c} d\left(x_{n-2}, x_{n-1}\right),
$$

for $n \geq 2$. Thus,

$$
d\left(x_{n}, x_{n+1}\right) \leq \eta d\left(x_{n-2}, x_{n-1}\right) .
$$

Hence, combining Case 1-3, it follows that

$$
d\left(x_{n}, x_{n+1}\right) \leq \eta A_{n}
$$

where

$$
A_{n}=\max \left\{d\left(x_{n-2}, x_{n-1}\right), d\left(x_{n-1}, x_{n}\right)\right\}, n \geq 2 .
$$

Following [11, by induction for $n \geq 2$, it follows that

$$
d\left(x_{n}, x_{n+1}\right) \leq \eta^{[n / 2]} A_{2},
$$

where

$$
A_{2}=\max \left\{d\left(x_{0}, x_{1}\right), d\left(x_{1}, x_{2}\right)\right\} .
$$

Herein, $[n / 2]$ imply the greatest integer not exceeding $n / 2$.

Now, for $m>n>N$,

$$
d\left(x_{n}, x_{m}\right) \leq \sum_{k=N}^{\infty} d\left(x_{k}, x_{k-1}\right) \leq \frac{2 \eta^{[n / 2]}}{1-\eta} A_{2} .
$$

Therefore $\left\{x_{n}\right\}$ is Cauchy sequence. By virtue of the fact that $\left\{x_{n}\right\} \subset H$ for all $n$ and $H$ is closed,

$$
x_{n} \rightarrow z \text { as } n \rightarrow \infty
$$

From the property $(L)$ and (8), we have

$$
\begin{aligned}
d\left(x_{k_{n}+1}, g z\right)= & d\left(g x_{k_{n}}, g z\right) \\
\leq \quad & a d\left(x_{k_{n}}, z\right)+b\left[d\left(x_{k_{n}}, g x_{k_{n}}\right)+d(z, g z)\right] \\
& +c\left[d\left(x_{k_{n}}, g z\right)+d\left(z, g x_{k_{n}}\right)\right] \\
\leq \quad & a\left[d\left(x_{k_{n}}, g x_{k_{n}}\right)+d\left(x_{k_{n}+1}, z\right)\right] \\
& +b\left[d\left(x_{k_{n}}, g x_{k_{n}}\right)+d\left(z, x_{k_{n}+1}\right)+d\left(x_{k_{n}+1}, g z\right)\right] \\
& +c\left[d\left(x_{k_{n}}, g x_{k_{n}}\right)+d\left(x_{k_{n}+1}, g z\right)+d\left(z, x_{k_{n}+1}\right)\right] .
\end{aligned}
$$

This yields,

$$
\begin{aligned}
(1-b-c) d\left(x_{k_{n}+1}, g z\right) & \leq(a+b+c) d\left(x_{k_{n}}, g x_{k_{n}}\right)+(a+b+c) d\left(z, x_{k_{n}+1}\right) \\
& \Rightarrow \\
d\left(x_{k_{n}+1}, g z\right) & \leq \eta\left[d\left(x_{k_{n}}, g x_{k_{n}}\right)+d\left(z, x_{k_{n}+1}\right)\right]
\end{aligned}
$$

which, due to (16) and taking the limit $n \rightarrow \infty$ infer that $\left\{x_{k_{n}}\right\}_{n=1}^{\infty} \rightarrow g z$ as $n \rightarrow \infty$. From (17), we conclude that $z=g z$.

Note that the uniqueness of $w$ simply follows by (8). 
Let $M=\left\{0, \frac{1}{7}, 1,2\right\}$ with recpect to metric $d(x, y)=|x-y|$, let $H=\{0,1,2\}$, and let $g x=0$ for all $x \in\{0,1\}$ and $g x=\frac{1}{7}$ for all $x \in\{2\}$ and $E(G)=\left\{(0,0),(0,1),(0,2),\left(2, \frac{1}{7}\right),(2,2),\left(\frac{1}{7}, \frac{1}{7}\right)\right\}$. Note that $G$ is weakly connected graph and $(M, d, G)$ is the property $(L)$. Further, it is easy to check that $g$ is (well) defined on $M$ with graph $G_{H}$. In fact, the subgraph $G_{H}$ of $G$ induced by $H$ has the set of vertices $E\left(G_{H}\right)=\{(0,1),(0,2),(2,2),(0,0),(1,1)\}$. In view of $[6]$, the edges $(0,2),(2,2)$ have to be ignored and for the remaining edges we get that

$$
(g 0, g 1)=(g 0, g 0)=(g 1, g 1)=(0,0) \in E\left(G_{H}\right) .
$$

Moreover, $g$ is a $G_{H}-$ Hardy-Rogers contraction on $H$. Indeed, we have 3 possible cases:

Case1. Let $x=0$ and $y=2$, then condition (8) reduces to

$$
|g 0-g 2|=\frac{1}{7} \leq 2 a+\frac{13}{7} b+\frac{15}{7} c .
$$

If we take $a=\frac{1}{10}, b=\frac{7}{260}$ and $c=\frac{7}{300}$, above inequality is obvious.

Case2. Let $x=1$ and $y=2$, then condition (8) reduces to

$$
|g 1-g 2|=\frac{1}{7} \leq a+\frac{20}{7} b+\frac{15}{7} c .
$$

If we take $a=\frac{1}{10}, b=\frac{7}{40}$ and $c=\frac{7}{30}$, above inequality is obvious.

Case 3. The condition (8) holds for the rest of the edges of $E\left(G_{H}\right)$.

Further Rothe's baundary condition is satisfied, as $\partial H=\{0,1,2\}$ and hence $g(\partial H)=\left\{0, \frac{1}{7}\right\} \varsubsetneqq H$. In the end, since we also have $H_{g}=\{0\} \neq \emptyset$, all hypotheses in Theorem 2 are not satisfied, however 0 is unique fixed point of $g$.

As in 11, 12, we relocated the property $(L)$ of the tripled $(M, d, G)$ by a suitable condition.

Let $(M, d, G)$ be a Banach spaces involving a simple directed and weakly connected graph $G$. Let $H$ be a nonempty closed of $M$ and $g: H \rightarrow M$ be a $G_{H}$-Hardy-Rogers contraction on $H$. If $H_{g}:=\{x \in \partial H:(x, g x) \in E(G)\} \neq$ $\emptyset, g$ is orbitally $G$-continuous and $g$ satisfies Rothe's baundary condition $g(\partial H) \subset H$, then $g$ has a fixed point in $H$.

Proof. Verbatim of the proof of Theorem 2, we conclude that the iterative sequence $\left\{x_{n}\right\}$ is Cauchy and converges to $z \in M$. Since $\left(g^{n} x_{0}, g^{n+1} x_{0}\right) \in E(G)$, for all $n$ and $g$ is orbitally $G$-continuous, we get that $z=\lim _{n \rightarrow \infty} g\left(g^{n} x_{0}\right)=$ $g z$. In other words, $z=g z$. Suppose that $t$ is another fixed point of $g$. Running a similar method like in Theorem 2. we have $z=t$.

(i) In Theorem 2 and Theorem 2, if we take $b=c=0$, we obtain Theorem 3.1 and Theorem 3.2 in [11, respectively.

(ii) In Theorem 2 and Theorem 2, if we take $a=c=0$, we obtain Theorem 2.1 and Theorem 2.2 in [12], respectively.

(iii) In Theorem 2 and Theorem 2 if we take $a=b=0$, we obtain Theorem 2 and Theorem 3 in [13, respectively.

(iv) In Theorem 2 and Theorem 2 , if we take $c=0$, we obtain the existence and uniqueness results for non-self Reich $G$-contractions in a Banach space with a graph.

Our theorems enhance and generalize fixed point theorems for some non-self contractions on Banach spaces involving a graph in Berinde \& Păcurar (2015), Balog \& Berinde (2016) and Balog et al. (2016). Within the future scope of the idea, reader might point out the existence and uniqueness of the fixed point point theorem for a Hardy-Rogers multi-valued contractions on a complete metric space via the $g$-graph preserving condition.

\section{Competing interests}

The author declare that she has no competing interests.

The author is thankful to the referees for their valuable comments and suggestions to improve this paper.

\section{References}

[1] Jachymski, J.: The contraction principle for mappings on a metric space with a graph. Proc. Amer. Math. Soc. 136, 1359-1373 (2008).

[2] Beg, I, Butt, AR, Radojević, S.: The contraction principle for set valued mappings on a metric space with a graph. Comput. Math. Appl. 60, 1214-1219 (2010). 
[3] Bojor, F.: Fixed point theorems for Reich type contractions on metric space with a graph. Nonlinear Anal. TMA 75 (9), 3895-3901 (2012).

[4] Öztürk, M, Girgin, E.: Some fixed point theorems for generalized contractions in metric spaces. Caspian Journal of Mathematical Sciences. 4(2)(2015), 257-270.

[5] Klanarong and Suantai: Coincidence point theorems for some multi-valued mappings in complete metric spaces endowed with a graph. Fixed Point Theory Appl. 2015, 129 (2015).

[6] Chifu, C, Petrusel, G.: New results on coupled fixed point theorem in metric space endowed with a directed graph. Fixed Point Theory Appl. 2014, 151 (2014).

[7] Suantai et al.: Common coupled fixed point theorems for $\theta-\psi$-contractions mappings endowed with a directed graph. Fixed Point Theory Appl. 2015, 224 (2015).

[8] Oztürk and Girgin: Some fixed point theorems and common fixed point theorems in metric space involving a graph. Bangmod Int. J. Math.\& Comp. Sci. vol.1, no.1,2015: pages 172-182.

[9] Yolacan, E, Kır, M.: New results for $\alpha$-Geraghty type contractive maps with some applications. Gazi University Journal of Science. 29(3): 651-658 (2016).

[10] Yolacan, E, Kızlltunc, H, Kır, M.: Coincidence point theorems for $\varphi-\psi$-contraction mappings in metric spaces involving a graph. Carpathian Math. Publ. vol 8, no:2 (2016). doi: 10.15330/cmp.8.2.251-262

[11] Berinde, V, Păcurar, M.: The contraction principle for non-self mappings on Banach spaces endowed with a graph. J. Nonlinear Convex Anal. Vol 16, no:9, 2015, 1925-1936.

[12] Balog, L, Berinde, V.: Fixed point theorems for non-self Kannan type contractions in Banach spaces endowed with a graph. Carpathian J. Math. 32 (2016), no: 293-302.

[13] Balog, L, Berinde, V, Păcurar, M.: Approximating fixed points of non-self contractive type mappings in Banach spaces endowed with graph. An. Şt. Univ. Ovidius Constanta. 2016. 24 (2), 27-43. 10.1515/auom-2016-0026.

[14] Berinde, V, Păcurar, M.: Fixed point theorems for non-self single valued almost contractions. 2013. Fixed Point Theory. 14(2), 301-312.

[15] Bojor, F.: Fixed points of Kannan mappings in metric spaces endowed with a graph. An. Şt. Univ. Ovidius Constanta. 2012. 20 (1), 31-40.

[16] Bojor, F, Tilca, M.: Fixed point theorems for Zamfirescu mappings in metric spaces endowed with a graph. Carpathian J. Math. 2015. 31(3), 297-305.

[17] Phon-on, A, Same-Ae, A, Makaje, N, Riyapan, P. Reich type weak contractions on metric spaces endowed with a graph. Fixed Point Theory Appl. 2015: 57.

[18] Banach, S.: Sur les operations dans les ensembles abstraits et leur applications aux equations integrales. Fundam. Math. 3, 133-181 (1922).

[19] Hardy, GE, Rogers, TD.: A generalization of a fixed point theorem of Reich. Can. Math. Bull. 16, 201-206 (1973).

[20] Ran, ACM, Reurings, MCB.: A fixed point theorem in partially ordered sets and some application to matrix equations. Proc. Amer. Math. Sco. 132 (5), 1435-1443.

[21] Edelstein, M.: An extension of Banach's contraction principle. Proc. Amer. Math. Sco. 12(1), 7-10. 